\section{Rituximab in the treatment of extensive and refractory subacute cutaneous lupus erythematosus*}

Mariana Álvares Penha ${ }^{1}$

Ricardo da Silva Libório ${ }^{1}$

Hélio Amante Miot ${ }^{1}$

\section{DOI: http:/ /dx.doi.org/10.1590/abd1806-4841.20187561}

\section{Dear Editor,}

Systemic lupus erythematosus (SLE) is an autoimmune disease with multiple clinical manifestations, such as renal, hematological, neurological, and cutaneous. Acute cutaneous lupus erythematosus (ACLE), subacute cutaneous lupus erythematosus (SCLE), and chronic cutaneous lupus erythematosus (CCLE), which includes discoid lupus erythematosus (DLE) and lupus erythematous panniculitis, are among the classic skin manifestations, present in $80 \%$ of SLE cases.

Photoprotection, antimalarials, dapsone, thalidomide, and corticosteroids are the treatments of choice for cutaneous lupus erythematosus (CLE). Immunosuppressive drugs, such as azathioprine, methotrexate, and cyclosporine, may also be used to control the disease. However, some cases are refractory to such treatments. The anti-CD20 monoclonal antibody rituximab (RTX) has been used as a therapeutic option in refractory cases with good results, mainly in patients with SCLE and severe extracutaneous forms of SLE. ${ }^{1}$

We aim to demonstrate a case of SCLE, refractory to conventional therapy, that was successfully treated with RTX and to review the literature of SCLE cases treated with RTX.

A 31-year-old female, brown-skinned, hypertensive and smoker, has been attending our outpatient clinic since 2002 due to SLE. She presents SCLE, photosensitivity, lymphopenia $(456 / \mathrm{ml})$, antinuclear antibodies (ANA) 1/640 with a homogenous pattern, and other immunological alterations (anti-Sm, anti-RNP, anti-Ro). No signs of renal, neurological or pulmonary impairment have been detected.

Physical examination revealed violaceous, erythematous, annular, exulcerated, and scaly plaques, mainly on sun-exposed areas, and labial mucous damage with exulcerated plaques (Figure 1).

\footnotetext{
Received 12 June 2017.

Accepted 17 September 2017.

* Work conducted at the Departament of Dermatology and Radiotherapy, Faculdade de Medicina de Botucatu, Universidade Estadual Paulista (FMB-Unesp), Botucatu (SP), Brazil.

Financial support: None.

Conflict of interests: None.

1 Departament of Dermatology and Radiotherapy, Faculdade de Medicina de Botucatu - Universidade Estadual Paulista (FMB-Unesp), Botucatu (SP), Brazil.
}

MAILING ADDRESS:

Hélio Amante Miot

E-mail: heliomiot@gmail.com

C 2018 by Anais Brasileiros de Dermatologia

(cC) BY-NC
The patient had previously been treated with azathioprine (150mg/day), hydroxychloroquine (400mg/day), thalidomide (100mg/day), dapsone (100mg/day), prednisone (0.5 to $1 \mathrm{mg} / \mathrm{kg} /$ day), and monthly pulse therapy with methylprednisolone (21 cycles) without complete control of the disease.

As an attempt of treatment, RTX was started at a weekly dose of $375 \mathrm{mg} / \mathrm{m}^{2}$ for 4 weeks, followed by maintenance doses every 6 months. Intravenous methylprednisolone $(100 \mathrm{mg})$ was also given with each RTX dose. One month after the first dose, the patient showed significant improvement with a complete and continuous control of the disease, reducing her disease activity index (SLEDAI) from 18 to 4.

Hydroxychloroquine, thalidomide, and dapsone doses were maintained and oral prednisone was gradually tapered without flares throughout a 12-month follow-up (Figure 2).

RTX is an anti-CD20 monoclonal antibody approved for treatment of B-cell lymphomas and rheumatoid arthritis. However, studies have shown its effectiveness in treating autoimmune skin disorders, such as pemphigus vulgaris, pemphigus foliaceus, vasculitis, and SLE, specially SCLE. RTX is an immunosuppressive drug that enables the gradual tapering of oral corticosteroids, thus reducing the side effects caused by its chronic use. ${ }^{2}$
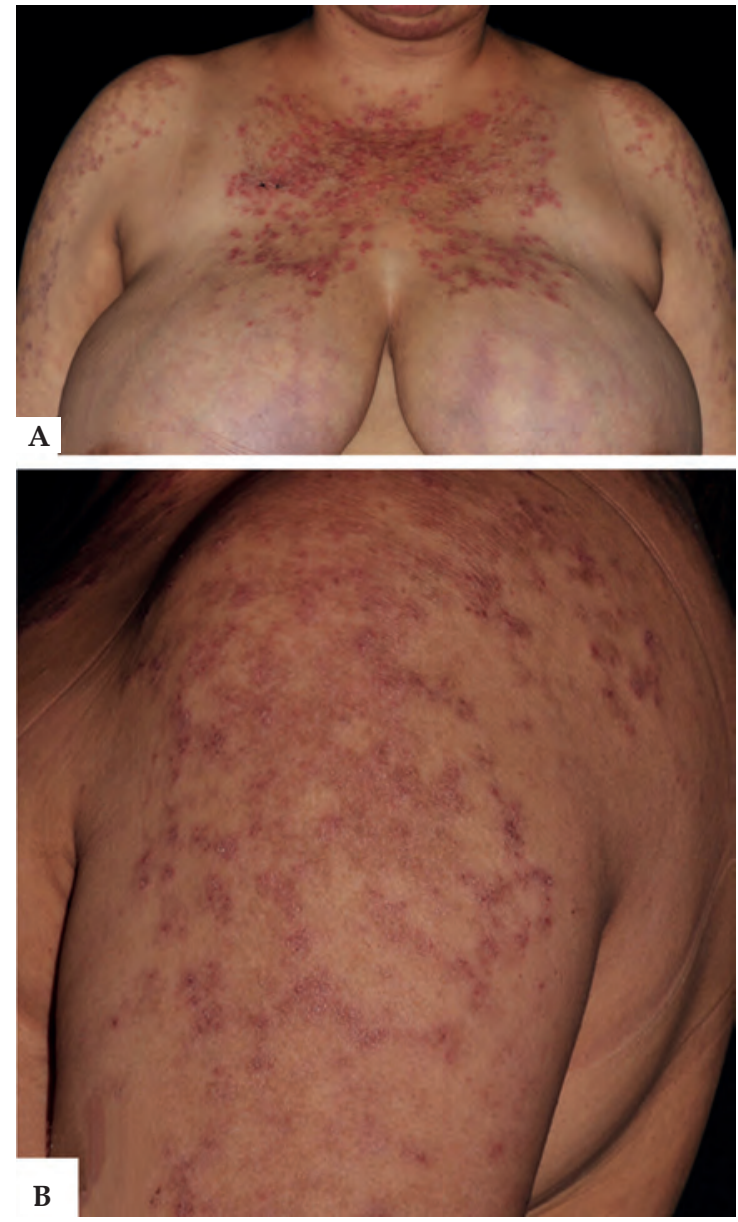

FIGURE 1: Sun-exposed areas with erythematous, annular, exulcerated, and scaly plaques before treatment with rituximab (RTX) 


\begin{tabular}{|c|c|c|c|c|c|}
\hline Sex/age & Therapy scheme & Therapeutic response & Maintenance therapy & Relapse & Previous therapy \\
\hline $\mathrm{F} / 402$ & $\begin{array}{l}\text { 2x 1g RTX + 100mg IV methyl- } \\
\text { prednisolone (every } 2 \text { weeks) }+ \\
750 \mathrm{mg} \text { of cyclosporine after first } \\
\text { infusion }\end{array}$ & Little & Not specified & None & $\begin{array}{l}\text { HCQ, Pred, MTX, } \\
\text { MMF }\end{array}$ \\
\hline $\mathrm{F} / 742$ & $\begin{array}{l}\text { 2x 1g RTX + 100mg IV methyl- } \\
\text { prednisolone (every } 2 \text { weeks) + } \\
750 \mathrm{mg} \text { of cyclosporine after first } \\
\text { infusion }\end{array}$ & $\begin{array}{l}\text { Complete } \\
\text { remission }\end{array}$ & Not specified & None & Pred, Aza, MTX \\
\hline $\mathrm{F} / 65^{2}$ & $\begin{array}{l}\text { 2x 1g RTX + 100mg IV methyl- } \\
\text { prednisolone (every } 2 \text { weeks) }+ \\
750 \mathrm{mg} \text { of cyclosporine after first } \\
\text { infusion }\end{array}$ & Complete remission & Not specified & None & Pred, MMF \\
\hline $\mathrm{M} / 44^{3}$ & 2x 1g RTX (weekly dose) & Complete remission & None & None & Pred, MMF, HCQ, Aza \\
\hline $\mathrm{F} / 48^{4}$ & 4x 375mg/m² RTX (weekly dose) & Complete remission & $\begin{array}{l}1 \text { dose every } 8 \text { weeks } \\
\text { for two years }\end{array}$ & $\begin{array}{l}11 \text { months } \\
\text { after first dose }\end{array}$ & $\begin{array}{l}\text { HCQ, Pred, MTX, } \\
\text { dapsone, Aza }\end{array}$ \\
\hline $\mathrm{F} / 54^{5}$ & $4 \times 375 \mathrm{mg} / \mathrm{m}^{2}$ RTX (weekly dose) & Complete remission & $\begin{array}{l}2 \times 1 \text { g every } 2 \text { weeks } \\
\text { for one year }\end{array}$ & None & $\begin{array}{l}\text { HCQ, Pred, MMF, } \\
\text { thalidomide, IGIV, } \\
\text { etanercept }\end{array}$ \\
\hline $\mathrm{F} / 37^{5}$ & $4 \times 375 \mathrm{mg} / \mathrm{m}^{2}$ RTX (weekly dose) & Complete remission & $\begin{array}{l}2 \times 1 \text { g every } 2 \text { weeks } \\
\text { for one year }\end{array}$ & None & HCQ, Pred, Aza \\
\hline $\mathrm{F} / 28^{5}$ & 4x 375mg/m² RTX (weekly dose) & Partial remission & Not specified & None & HCQ, Pred \\
\hline
\end{tabular}

RTX: rituximab/ Pred: prednisone/ Aza: azathioprine/ MMF: mycophenolate mofetil/ HCQ: Hydroxychloroquine/ MTX: methotrexate / IGIV: intravenous immunoglobulins/ F: female/ M: male

Currently, there are only eight published cases of patients with refractory SCLE treated with RTX (Table 1)..$^{2-5}$ In all published cases, the SCLE patients selected to use RTX had otherwise been refractory to conventional therapy. Except for 1 case, the remaining 7 patients showed effective results with little side effects.

RTX doses varied from $1 \mathrm{~g}$ twice a week to $375 \mathrm{mg} / \mathrm{m}^{2}$ a week for 4 weeks. Maintenance doses, when used, varied from 2 months to 1 year. In the case we report here, the dose was similar to that used in the Australian study (weekly doses of $375 \mathrm{mg} / \mathrm{m}^{2}$ for 4 weeks). However, since the patient's condition improved significantly and there were no flares, maintenance doses were used every 6 months. ${ }^{4}$

Similar to most cases reported in the literature, our patient's condition improved one month after the first RTX dose, which enabled the gradual tapering of prednisone, thus reducing the side effects caused by its chronic use. Some studies reported flares after treatment started, but lesions were cleared after maintenance doses.

Although RTX has shown effective results in the treatment of SCLE, it has not been effective for cutaneous lesions of other forms of CLE, such as CCLE. This suggests that RTX is indicated for lupus manifestations involving Th2 cells, such as serositis, vasculitis, nephritis, ACLE and SCLE, which are mediated by B cells (CD20+).

Further studies are needed in order to recognize RTX as a therapeutic option for SCLE, even though independent results indicate that it is effective in the treatment of refractory SCLE. Data regarding dose / frequency of administration, drug tolerability, side effects, and long-term remission are still lacking given the small number of reported cases. $\square$
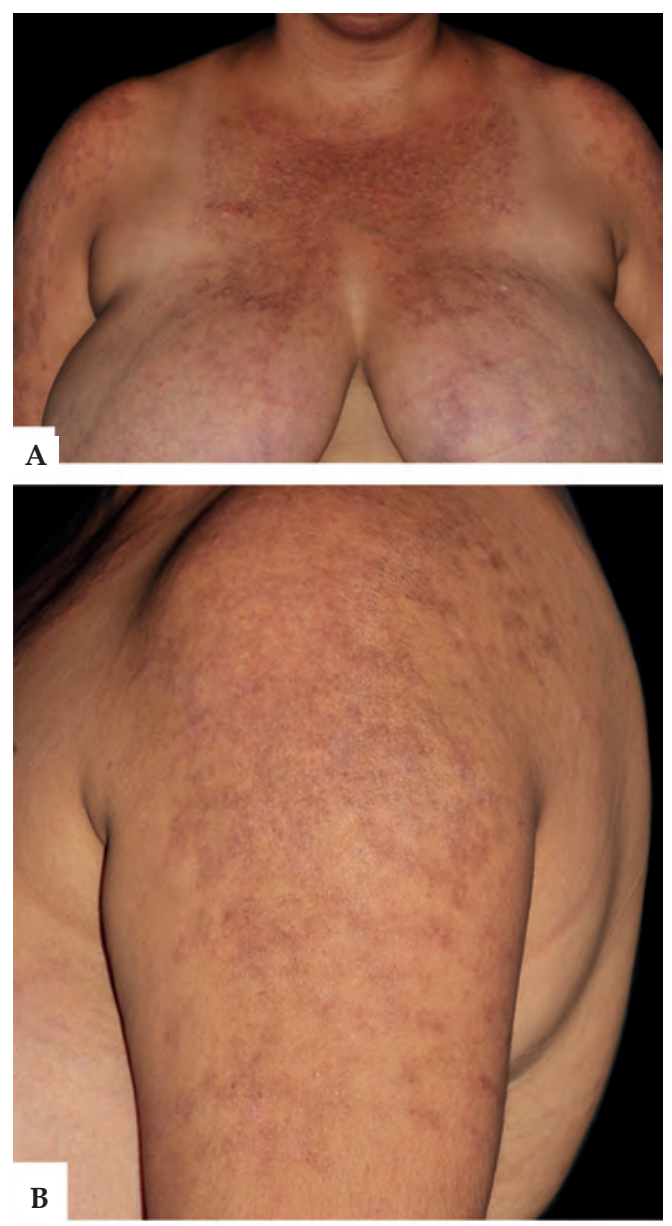

FigURA 2: Post-inflammatory hyperchromic patches 7 months after treatment with rituximab (RTX); absence of active lesions 


\section{REFERENCES}

1. Presto JK, Hejazi EZ, Werth VP. Biological therapies in the treatment of cutaneous lupus erythematosus. Lupus. 2017;26:115-8

2. Hofmann SC, Leandro MJ, Morris SD, Isenberg DA. Effects of rituximab-based B-cell depletion therapy on skin manifestations of lupus erythematosus--report of 17 cases and review of the literature. Lupus. 2013;22:932-9.

3. Uthman I, Taher A, Abbas 0, Menassa J, Ghosn S. Successful treatment of refractory skin manifestations of systemic lupus erythematosus with rituximab: report of a case. Dermatology. 2008;216:257-9.

4. Kieu V, O'Brien T, Yap LM, Baker C, Foley P, Mason G, et al. Refractory subacute cutaneous lupus erythematosus successfully treated with rituximab. Australas $\mathrm{J}$ Dermatol. 2009;50:202-6

5. Cieza-Díaz DE, Avilés-Izquierdo JA, Ceballos-Rodríguez C, Suárez-Fernández R. Refractory subacute cutaneous lupus erythematosus treated with rituximab. Actas Dermosifiliogr. 2012;103:555-7.

Mariana Álvares Penha

ORCID 0000-0001-6069-8015

Ricardo da Silva Libório

(iD) ORCID 0000-0003-4700-3973

Hélio Amante Miot

ORCID 0000-0002-2596-9294

How to cite this article: Penha MA, Liborio RS, Miot HA. Rituximab in the treatment of extensive and refractory subacute lupus erythematosus. An Bras Dermatol. 2018;93(3):467-9.

\section{Hydroxychloroquine ototoxicity in a patient with systemic lupus erythematosus*}

Mariana Rita de Novaes Fernandes ${ }^{1}$

Débora Bergami Rosa Soares ${ }^{1}$

Chan I Thien ${ }^{1}$

Sueli Carneiro ${ }^{1,2,3,4}$

DOI: http:/ /dx.doi.org/10.1590/abd1806-4841.20187615

Dear Editor,

We report the case of a 51-year-old female patient who presented with alopecia and erythematous atrophic plaques with some areas of hyperpigmentation on the face and scalp in 2006. At the time,

\footnotetext{
Received 04 September 2017.

Accepted 29 November 2017.

* Work CONDUCTED at Hospital Universitário Pedro Ernesto, Universidade do Estado do Rio de Janeiro (HUPE-UERJ), Rio de Janeiro (RJ), Brazil. Financial support: None.

Conflict of interests: None.

${ }^{1}$ Service of Dermatology, Hospital Universitário Pedro Ernesto, Universidade do Estado do Rio de Janeiro (HUPE-UERJ), Rio de Janeiro (RJ), Brazil.

2 Department of Medical Specialties, Faculdade de Ciências Médicas, Universidade do Estado do Rio de Janeiro (HUPE-UERJ), Rio de Janeiro (RJ), Brazil.

${ }^{3}$ Service of Dermatology, Hospital Universitário Clementino Fraga Filho, Universidade Federal do Rio de Janeiro (HUCFF-UFRJ), Rio de Janeiro (RJ), Brazil.

${ }^{4}$ Service of Rheumatology, Hospital Universitário Clementino Fraga Filho, Universidade Federal do Rio de Janeiro (HUCFF-UFRJ), Rio de Janeiro (RJ), Brazil.
}

MAILING ADDRESS:

Mariana Rita de Novaes Fernandes

E-mail: mari.ritafernandes@gmail.com

(C)2018 by Anais Brasileiros de Dermatologia she was diagnosed with chronic cutaneous lupus erythematous after a histologic report of a biopsy taken from the scalp, and started treatment with topical corticosteroids. In 2011, she developed fever, weight loss, fatigue and arthritis of the proximal and distal metacarpophalangeal joints, hands, elbows and shoulders. Hydroxychloroquine $5 \mathrm{mg} / \mathrm{kg} /$ day $(400 \mathrm{mg} /$ day) was commenced. Three years after regular use of the medication, she complained of tinnitus and bilateral hearing loss. Liminar tonal audiometry showed moderate neurosensory hearing loss on the left ear and mild to moderate on the right ear. The abnormalities found on the audiometry are suggestive of hydroxychloroquine toxicity. Antiphospholipid antibodies were negative, aiding in the exclusion of lupus erythematosus neurosensory dysacousia. The medication was discontinued, and four months later she still complains of tinnitus and dysacousia. She is undergoing clinical follow-up.

Hydroxychloroquine is a relatively safe drug, with uncommon side effects, except for retinopathy associated to the use in high doses. ${ }^{1}$ Drug-related ototoxicity is defined by a transient or permanent disturbance of the auditory and/or vestibular function induced by therapeutic substances. ${ }^{2}$ Many drugs have the potential of causing vestibulocochlear toxicity and, although the antimalarial activity of hydroxychloroquine is the same as the chloroquine sulfate, its toxic potential is significantly lower. ${ }^{2,3}$ Its derivatives, chloroquine and hydroxychloroquine, are widely used in connective tissue disease, adverse effects as cutaneous hyperpigmentation and retinopathy are known and usually monitored in those using these drugs. ${ }^{1,2}$

Quinine-induced ototoxicity is manifested by auditory and vestibular dysfunction; however, its exact mechanism is yet not well established. The drug is absorbed by the gastrointestinal tract and most of it is deposited in the tissues, with only a minimal portion excreted. ${ }^{2}$ Chloroquine builds up and remains selectively fixed to melanocytes, and high levels of the drug are present in the stria vascularis, retinal pigment, skin, hair follicle and endocrine glands. ${ }^{1}$ Ototoxicity is related to the destruction of the stereocilia in varying degrees, reducing the neuron population, altering the support structures, causing atrophy of the stria vascularis and potentially leading to ischemia. ${ }^{1}$ Melanin is present in the inner ear in highly vascular areas, thus, blood vessels are usually surrounded by melanocytes. In this context, it is believed that the buildup of chloroquine is responsible for a vascular injury and degenerative changes in the planum semilunatum and stria vascularis. These abnormalities of the epithelial tissues could result in an alteration of the structure of the endolymph, leading to damage of the cellular receptor. ${ }^{2}$ The buildup and long-term retention of antimalarial in melanocytes of the inner ear could explain the late onset of lesions and the relationship with elevated cumulative doses. ${ }^{2}$

The main symptoms associated to the use of antimalarials are tinnitus, neurosensory hearing loss and vertigo. Hearing loss is considered irreversible, with the report of some exceptions. ${ }^{2}$ Seçkin reports a case of ototoxicity with HCQ in a patient with rheumatoid arthritis who developed mild bilateral neurosensory dysacousia and tinnitus. After discontinuation of the treatment, she improved of the tinnitus and on the audiogram. 